\title{
Sleep Apnea Syndrome and Covid-19 Infection Correlation: Treated/Not Treated OSAS Patients Comparison
}

\author{
Francesca Francese $^{1^{*}}$, Giuliana Conte ${ }^{2}$ and Mario Ermani ${ }^{2}$ \\ ${ }^{1}$ Sleep Lab-Pederzoli Hospital, Italy \\ ${ }^{2}$ University of Padova, Italy
}

*Corresponding author: Francesca Francese, Sleep Lab-Pederzoli Hospital, Italy

\begin{abstract}
This work is intended to study respiratory function during sleep in positive Covid patients in order to evaluate the presence of sleep apnea syndrome as risk factor that predispose to Covid infection.

Data shows that the comorbidities in Covid patients are obesity, hypertension, diabetes and cardiovascular diseases [1].

The same comorbidities are mostly present in people suffering from sleep apnea syndrome [2].

OSAS syndrome causes fragmented sleep and cytokine production (IL-6, IL-17 and TNFa); consequently it represents a pro inflammatory condition [3].

The reason consists in snoring; the vibrations of the soft walls cause an inflammation of the upper airways, over time increasing inflammation, respiratory events increase.

We have studied 32 hospitalized Covid patients, at T0 time and T1 time, with sleep test considering some physiological parameters as airflow, thorax and abdominal movements, body position, snoring, heart rate and oxygen level in the blood. The device used for the study is Nox T3.

Studies are evaluated with manual analysis according to international guidelines with differences in the nature of apnoic and hipoapnoic events.

Seven patients have no comorbidity, 25 of them have comorbidities such as cardiovascular diseases, diabetes, respiratory diseases, upper airway obstruction. 27 patients are overweight or obese. Sleep test results at T0 time show that 4 patients are negative, 15 patients have mild OSAS, 5 patients have moderate OSAS and 8 patients have severe OSAS.
\end{abstract}

Three of negative patients have cardiovascular diseases.

We repeated sleep test at T1 time on 29 patients (2 patients died, 1 patients was absent). 1 patient is negative, 15 patients have mild OSAS, 7 patients have moderate OSAS, 6 patients have severe OSAS.

All variables studied such as BMI, age, supine time, hours sleep, oxygen saturation, respiratory rate, comorbidities, were not statistically significant. No correlation between the studied variables and the evolution of sleep apnea syndrome in Covid infection was found.

We also compared two groups of patients; 55 OSAS patients in ventilatory therapy (with Cpap) and 55 OSAS patients who refused the treatment.

Data shows no statistically significant differences between the two groups referring to Covid infection development independently from OSAS severity and independently from the number of comorbidity present in these patients; no significant differences was found between group with comorbidity and the other group without comorbidity. Also referring to the type of course (home, hospitalized, dead), no statistically significant difference was found.

16 of 110 patients known as OSAS developed Covid infection; $12 \%$ were treated patients and $16 \%$ were not treated patients, so $14 \%$ of all Known OSAS patients studied during February 2021.

Comparing this data with all the population of Veneto, it was found that the $7 \%$ had Covid infection. These percentages are significantly different.

Considering these percentages, OSAS could be a risk factor for Covid infection. 


\section{Introduction}

Obstructive sleep apnea (OSA) is a common disorder characterized by upper airway caliber reduction during sleep, habitual and persistent snoring, every night for at least six months and repetitive episodes of nocturnal breathing cessation, partial or complete, due to upper airway collapse; each pause can last for a few seconds to a few minutes and they happen many times a night. Hypopnea and apneas events make oxygen level decreasing, this is called hypoxemia $[4,5]$.

It causes cerebral, metabolic and cardiovascular diseases and some pathophysiological consequences such as respiratory effort and endothoracic negative pressure increased, sympathetic vegetative activation, sleep micro- awakening, intermittent hypoxemia, inflammatory processes [6]; it produces an alterated immunoregulation and respiratory instability during sleep [7].

From this point the idea of this study starts. Because of sleep apnea syndrome causes an upper airway inflammation [8,9], we ask if OSAS patients could take Covid infection easier, if there are variables that can affect the Apnea/Hypopnea index (AHI) at T1 time, if the presence of comorbidities affects the OSAS trend and if OSAS therapy is a protective factor for Covid infection.

\section{Materials and Methods}

First, we have considered 32 hospitalized positive Covid patients and we have studied their sleep at T0 and T1 time (Longitudinal Study-repeated measures). Sleep study has evaluated by nocturnal cardiorespiratory monitoring carried out over the course of one night.

The Nox T3 polygraph equipped with chest and abdominal belt, position sensor, snoring derived from the audio signal, plethysmography, night saturation and heart rate were also used.

Considering to the guidelines during Covid pandemic, the nasal cannula for airflow detection was not used. Flow analysis was performed from the $X$ flow lead allowed by the use of plethysmographic bands.

Plethysmographic bands were used to record thoracic abdominal movements, their advantages of which are listed below:

- They allow to measure the variation of the volumes below the bands

- In any position of the body they can measure the part of the chest and abdomen that is moving

- The signal obtained is very similar to that of the thermistor and reproduces inspiration and expiration

Studies are evaluated with manual analysis according to the international guidelines, with differentiation of the nature of not only apnoic but also hypoapnoic events.
Patients examinated was random patients with a mild clinical status at entry: it means they have maximum 1 liter of oxygen.

Therefore, only patients with clinical characteristics that presuppose a suspicion of the disease were not considered.

A database was filled considering various variables: age, death, gender, BMI at T0 time and T1 time, respiratory frequency during sleep at T0 and T1 time, average saturation at TO and T1 time, OSAS level at TO and $\mathrm{T} 1$ time, $\mathrm{AHI}$ at $\mathrm{TO}$ and $\mathrm{T} 1$ time.

It reminds that $\mathrm{AHI}$ is apnea and hypoapneas index/ hours of sleep. OSAS level should be determined just considering obstructive hypoapneas and apneas events.

We have 3 OSAS levels: if $\mathrm{AHI}$ is between 5-14 we have mild OSAS, between 15 and 29 moderate OSAS, > 30 severe OSAS.

Other variables were considered: OSAS supine position time at TO and T1 time and four kinds of comorbidities.

Comorbidity 1 represents upper airway obstructions such as nasal septal deviation, turbinate hypertrophy, tonsillar hypertrophy.

Comorbidity 2 represents cardiovascular diseases such as hypertension, atrial fibrillation, hypertensive cardiopathy.

Comorbidity 3 represents diabetes.

Comorbidity 4 represents respiratory diseases like asthma, BPCO, emphysema. Second, we have considered 110 known OSAS patients (Trasversal study); half of them in therapy, the other half not in therapy in order to observe how many of them had Covid infection based on therapy or non-therapy.

Various variables were examined: age, gender, therapy, Covid, course of the disease (home, hospitalized, death), AHI.

In the half OSAS patients in therapy the $\mathrm{AHI}$ is referring to the residual events under therapy.

In the other half ones the number of $\mathrm{AHI}$ is referring to the diagnostic AHI. In addition, referring to treated OSAS patients, we considered also hours of use of the therapy and the therapy compliance.

Compliance is defined by the number of hours per night and the percentage of nights in which the therapy is adequately used.

It is considered acceptable to use $>70 \%$ of the nights, good $>80 \%$ of the nights and excellent $>90 \%$ of the nights [10].

At the end, for each patient we have associated the presence of one or more comorbidities. 


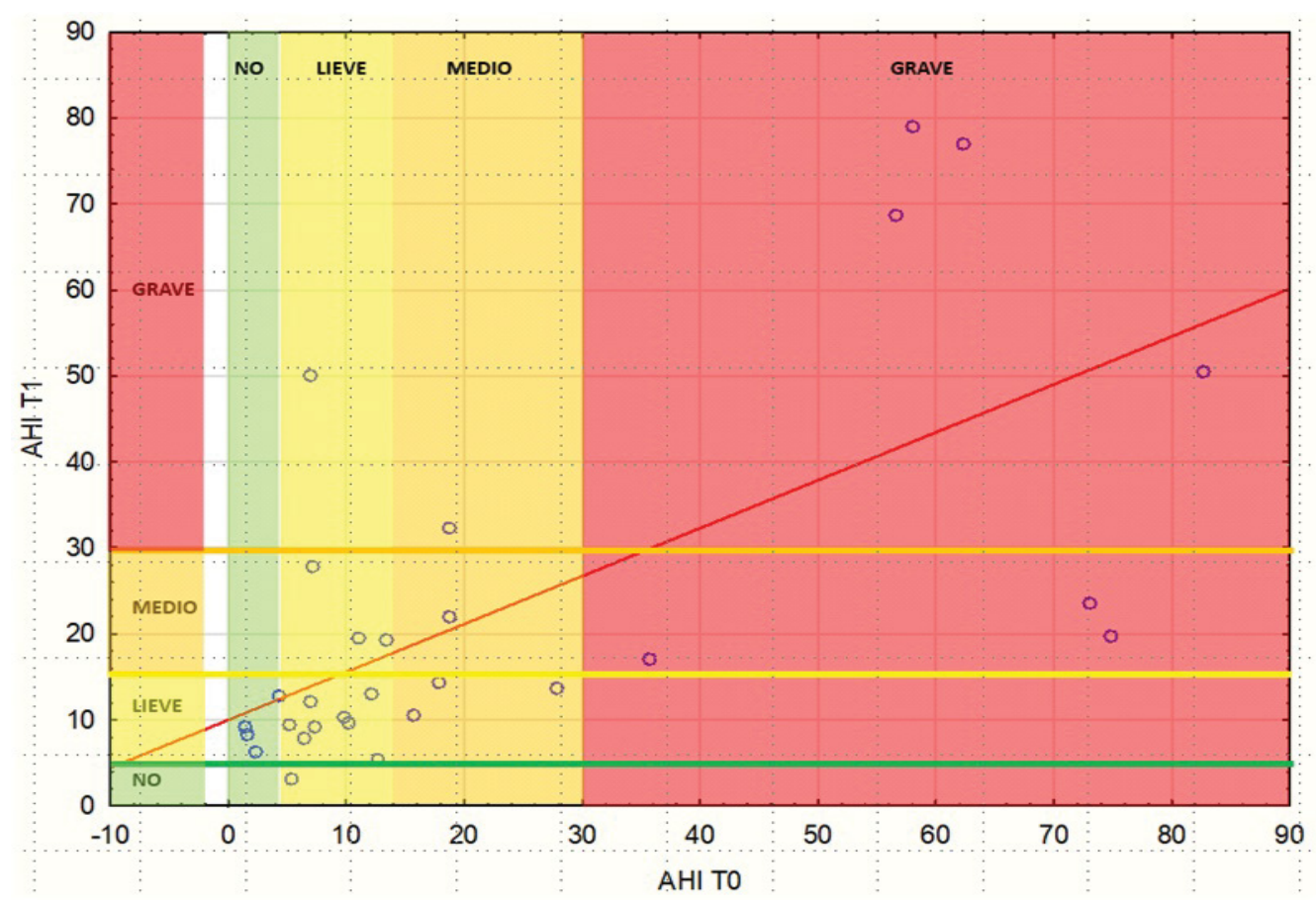

Figure 1: Linear correlation between $\mathrm{AHI} T \mathrm{TO}-\mathrm{AHI}$ T1 time.

Table 1: Correlation DB longitudinal.

\begin{tabular}{|c|c|c|c|c|c|c|}
\hline \multirow[t]{2}{*}{ Variable } & \multicolumn{6}{|c|}{$\begin{array}{c}\text { Correlations (DB longitudinal) marked correlations are significant at } \mathrm{p}<0.05000 \mathrm{~N}=29 \text { (Case wise } \\
\text { deletion of missing data) }\end{array}$} \\
\hline & ETA' & BMI T0 & FR s T0 & SAT M TO & AHI TO & T SUPINO TO \\
\hline \multirow[t]{2}{*}{ AHI T1 } & 0.2274 & 0.2470 & 0.2849 & -0.5274 & 0.6493 & -0.2173 \\
\hline & $p=0.236$ & $p=0.196$ & $p=0.134$ & $p=0.003$ & $p=0.000$ & $p=0.257$ \\
\hline
\end{tabular}

Comorbidity 1 represents upper airway obstructions such as nasal septal deviation, turbinate hypertrophy, tonsillar hypertrophy.

Comorbidity 2 represents cardiovascular diseases such as hypertension, atrial fibrillation; hypertensive cardiopathy.

Comorbidity 3 represents diabetes.

Comorbidity 4 represents respiratory diseases like asthma, BPCO, emphysema.

\section{Statistical Analysis}

The Student T test, Mann-Whitney $U$ test and Chi Square test were used for normal, ordinal and nominal variables respectively. Paired $T$ test was used for repeated measures. Correlation between variables was tested using simple and multivariate linear regression model. Logistic regression model was applied to find the true independent predictors for develop a severe OSA, considering as dependent variable the dichotomized OSA (severe/other), and as independent variables those found to be significant in monovariate analysis. ROC curve and the Youden method were applied to find the cut-off of the AHI. Significance level was set at $p<0.05$. The statistical packages STATISTICA V.13 and SPSS V24 were used.

\section{Results}

Study results referring to hospitalized Covid patients, show that $87 \%$ of hospitalized Covid patients have OSAS at T0 and $93 \%$ of hospitalized Covid patients have OSAS at $\mathrm{T} 1$.

Referring to linear correlation (Figure 1), the relation between OSAS level at TO time and OSAS level at T1 time is quite linear in the first period for mild-moderate OSAS level. For severe OSAS level the prediction does not work anymore.

The interesting fact is that the line behaves independently from the considered variables.

Because of $\mathrm{AHI}$ at $\mathrm{t} 1$ determines the classification of patients, we could ask ourselves what are the variables correlated with AHI at time t1 (Table 1).

From a linear correlation point of view, two are correlated: oxygen saturation and AHI. Better is the situation at the beginning in terms of oxygen saturation and OSAS level; better is the situation at T1 time. There are extreme situations in which linearity is not preserved at all (Figure 2).

Referring to known OSAS patients, there was not significant association between comorbidity and Covid 

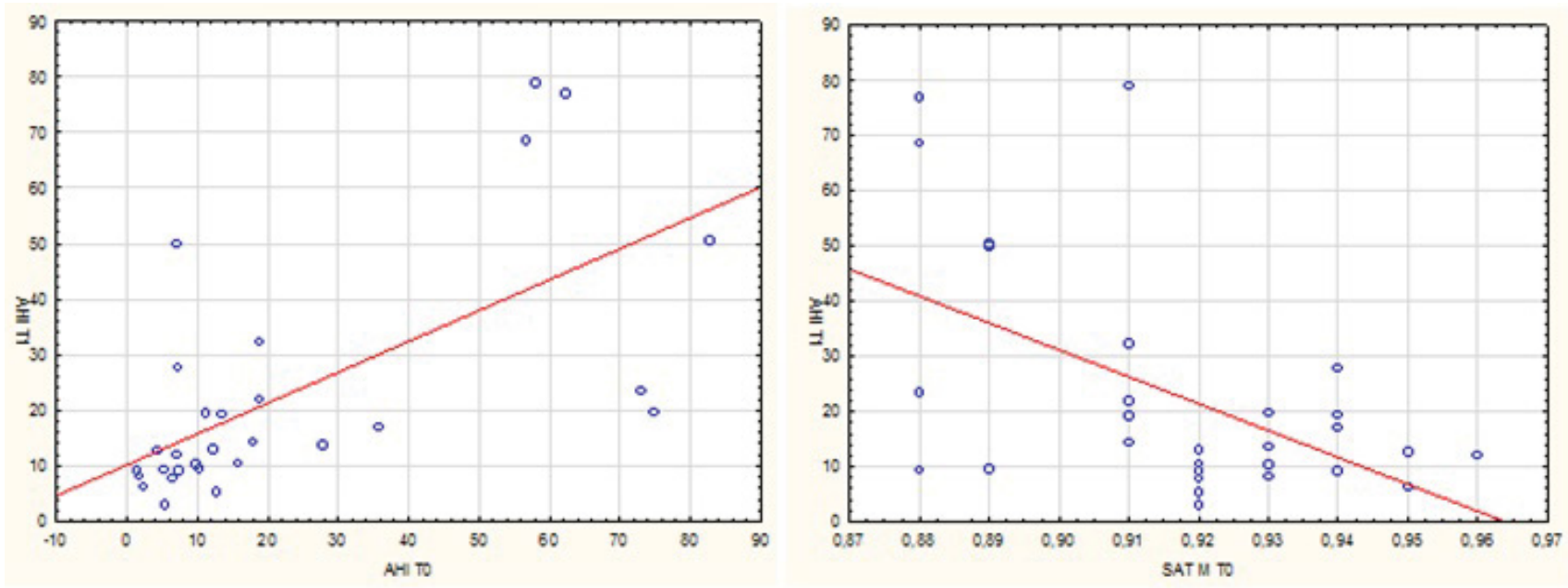

Figure 2: Linear correlation between $\mathrm{AHI}$ at $\mathrm{t} 1$ time and oxygen saturation at $\mathrm{T} 0$ time.

Table 2: Summary frequency table - Diabetes.

\begin{tabular}{|l|l|l|l|l|}
\hline & \multicolumn{5}{|c|}{ Summary Frequency Table } \\
\hline & Diabetes & $\begin{array}{l}\text { Covid } \\
\text { Yes }\end{array}$ & $\begin{array}{l}\text { Covid } \\
\text { No }\end{array}$ & Row Totals \\
\hline Count & 0 & 13 & 80 & 93 \\
\hline Row Percent & & $13.98 \%$ & $86.02 \%$ & 17 \\
\hline Count & 1 & 3 & 14 & \\
\hline Row Percent & & $17.65 \%$ & $82.35 \%$ & \\
\hline
\end{tabular}

Not significant: $p=0.69$

Table 3: Summary frequency table - Asthma, BPCO, Emphysema.

\begin{tabular}{|l|l|l|l|l|}
\hline & \multicolumn{5}{|c|}{ Summary Frequency Table } \\
\hline & Asthma-BPCO-Emphysema & $\begin{array}{l}\text { Covid } \\
\text { Yes }\end{array}$ & $\begin{array}{l}\text { Covid } \\
\text { No }\end{array}$ & Row Totals \\
\hline Count & 0 & 15 & 86 & 101 \\
\hline Row Percent & & $14.85 \%$ & $85.15 \%$ & 9 \\
\hline Count & 1 & 1 & 8 & \\
\hline Row Percent & & $11.11 \%$ & $88.89 \%$ & 110 \\
\hline Count & ALL Grps & 16 & 94 & 9 \\
\hline
\end{tabular}

Not significant: $p=0.76$.

Table 4: Summary frequency table - course of Covid.

\begin{tabular}{|l|l|l|l|l|l|l|}
\hline & \multicolumn{5}{|c|}{ Summary Frequency Table } \\
\hline & Therapy & $\begin{array}{l}\text { Course } \\
\text { domicile }\end{array}$ & $\begin{array}{l}\text { Course } \\
\text { hospitalized }\end{array}$ & $\begin{array}{l}\text { Course } \\
\text { dead }\end{array}$ & $\begin{array}{l}\text { Row } \\
\text { Totals }\end{array}$ \\
\hline Count & Yes & 5 & 2 & 0 & 7 \\
\hline Row Percent & & $71.43 \%$ & $28.57 \%$ & $0.00 \%$ & 9 \\
\hline Count & No & 7 & 1 & 1 & $11.11 \%$ & \\
\hline Row Percent & & $77.78 \%$ & $11.11 \%$ & 1 & 16 \\
\hline Count & All Grps & 12 & 3 & 1 & 9 \\
\hline
\end{tabular}

development. For each comorbidity, $\mathrm{p}$ Value is $>0.05$

Essentially there is not statistically significant difference in comorbidity distribution and in comorbidity number simultaneously present in the two groups. $\mathrm{P}=$ 0.97 (Table 2, Table 3, Table 4 and Table 5).

Also referring to the type of course (domicile, 
Table 5: Summary frequency table - therapy-Covid.

\begin{tabular}{|l|l|l|l|l|}
\hline & Therapy & $\begin{array}{l}\text { Covid } \\
\text { Yes }\end{array}$ & $\begin{array}{l}\text { Covid } \\
\text { No }\end{array}$ & $\begin{array}{l}\text { Row } \\
\text { Totals }\end{array}$ \\
\hline Count & Yes & 7 & 48 & 55 \\
\hline Row Percent & & $12.73 \%$ & $87.27 \%$ & \\
\hline Count & No & 9 & 46 & 55 \\
\hline Row Percent & & $16.36 \%$ & $83.64 \%$ & \\
\hline Count & All Grps & 16 & 94 & 110 \\
\hline
\end{tabular}

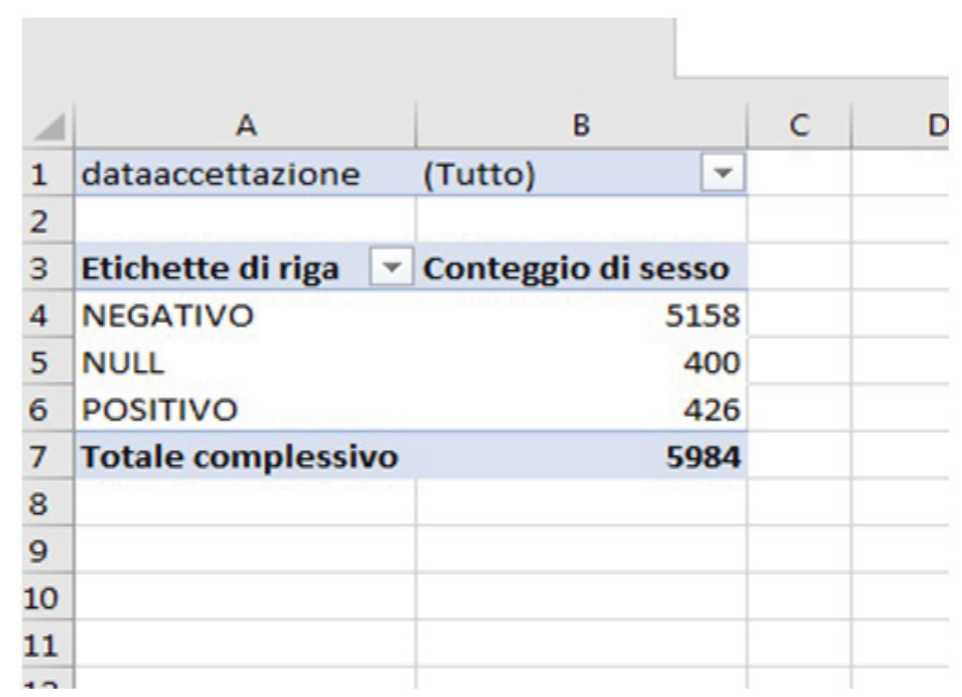

Figure 3: Veneto data collection during February 2021.

hospitalized, dead), no statistically significant difference was found $(p=0.49)$.

No statistically significant difference was found $(p=$ 0.59 ) between the two groups (with and without Cpap therapy) referring to Covid infection.

In particular on 110 OSAS patients, $12 \%$ of treated OSAS patients develop in Covid vs. $16 \%$ of not treated OSAS patients (Table 4, Table 5 and Figure 3).

Even if there is not a statistically significance difference, this data shows something and the comparison with general population in Veneto during February 2020 shows that $7 \%$ of general population had Covid vs. $14 \%$ of OSAS population.

\section{Discussion}

\section{Study repeated measures}

A population of hospitalized Covid subjects was considered and the different degrees of OSAS present were evaluated.

We observed what happens at time t0 and at time $\mathrm{t} 1 \mathrm{in}$ order to see if the variables considered vary over time: only breathing rate in sleep and percentage supine time change.

It is strange to note that neither saturation nor $\mathrm{AHI}$ changes over time.
Reason due to the fact that, on average, these two variables have remained the same but depending on the group considered, someone fared better someone worse.

The table regarding the change in OSAS severity between $\mathrm{T} 0$ and $\mathrm{T} 1$ shows that the 4 patients normal at t0, all had mild OSAS at T1.

The 13 patients who were mild at to, at $\mathrm{t} 1$ three became medium, 8 remained mild, one got better, one got worse.

Of those who had medium OSAS at t0, which were 5 , one remains the same, one gets worse and 3 becomes mild. Of the 7 severe at T0, 3 improve and 4 remain unchanged.

The interesting thing to see is whether there are predictive values in the trend. We measure the severity degree of OSAS by virtue of $A H I$, so since $A H I$ at $t 1$ determines the classification of patients, we could ask ourselves what are the variables correlated with $\mathrm{AHI}$ at time t1 (age, respiratory rate, $\mathrm{BMI}$, supine $\mathrm{t}, \mathrm{AHI}$ ).

From a linear correlation point of view, two are correlated: oxygen saturation and AHI. The higher the saturation, the lower it will be $\mathrm{AHI}$ : When the saturation increases AHI decreases. In fact, the correlation index is inverse. 
We see that there is a beautiful relationship with Ahi at t0.

In the univariate correlation graph we put $\mathrm{AHI}$ To in $\mathrm{x}$ and $\mathrm{AHI} \mathrm{t} 1$ in $\mathrm{y}$. The dots around the straight line are there enough up to $\mathrm{AHI}$ t0 to 30 .

The farther you go, that is, the worse the initial situation is, the more the opposite of everything happens: In these cases the straight line no longer works, there is something strange that it changes.

As for the inverse relationship between oxygen saturation at time t0 and $\mathrm{AHI}$ at time $\mathrm{t} 1$ : The more the saturation increases, the more Ahi is reduced, but there are extreme situations in which linearity is not preserved at all.

Predicting something in terms of the severity of the OAS (it will be mild, moderate,) is impossible because the cases are reduced.

So the idea was to predict if there will be a serious challenge, because it stands out from all the rest. Hence, severe OSAS at $\mathrm{t} 1$ or non severe OSAS at $\mathrm{t} 1$ was taken as dichotomization.

Then all the serious ones were put in one category and the others in another category.

In this way we can see that at to there were 7 severe ones and at $\mathrm{t} 1$ the serious ones were 6 : There is agreement. Of those 7 serious, 4 are left and 3 are not serious, vice versa of those that at $t 1$ were serious 4 were already there before and 2 are new.

Multivariate logistic regression showed that only Ahi at t0 was an independent predictive factor.

Then we calculated the ROC curve: the AUC (area under the curve) was 0.81 with confidence limits from 0.6 to 1 : That tells us that 0.81 would be a fair predictive capacity but it is very variable because we have a relative small sample size.

Using the Youden method the cutoff of $\mathrm{AHI}$ at tO as identified as 18.75 .

Considering the computed cut-off, a $2 \times 2$ table was created for correct allocation of cases. Sensitivity and specificity were good (about $80 \%$ ) and the OR was 23 (this means that a patients with $\mathrm{AHI}$ t0 greater than 18.75 have 23 times the probability of a sever OSA at t1, than a patients with $\mathrm{AHI}$ at to lower than 18.75) with large confidence limits due to the relatively small sample size.

Nevertheless $\mathrm{AHI}$ at TO is a true risk factor because both the left and right limits are higher than the value 1 which differentiates the risk factors from the protective factors.

There were five cases of erroneous allocation. As for the table of erroneous forecasts.
Case A: Those expected to have severe dares but do not have it.

There is a certain forecasting capacity but there is uncertainty because a bit of everything happens.

What connects $\mathrm{AHI}$ t1 to everything else? The $\mathrm{BMI}$ is not associated. It cannot be predicted without significant association.

Variations of BMI and supine time percentage are justified for the single patient but not in the group.

The trend of the OSAS over time is not influenced by any variable. Two databases have been created.

One concerns the longitudinal study-repeated measure which tries to answer the question of what the OSAS trend over time is.

It is an attempt to predict the trend of OSAS in a group of patients hospitalized for Covid.

Basically we ask ourselves if we can predict some clarity for a certain group of people with a cut off that has been identified 18 knowing that we have $80 \%$ sensitivity and specificity.

It is important to underline that the cases of severe OSAS are not many so the estimate is inaccurate.

There is therefore a certain predictive capacity but many cases are out of the question because the trend of the AHI cannot be explained by other factors present at the same time.

They were not significantly correlated in multivariate analysis as the sample size is not sufficient.

The real engine is $\mathrm{AHI}$ because it is on this that the definition of the OSAS levels is based.

Attempts to relate improvements to worsening.

But a person that ranges from mild to severe is different from another that ranges from mild to moderate.

Thus severe to mild and severe to moderate.

So even making a series of improvements on the same category worsened/improved, the data is too conflicting, it is impossible to predict something given the enormous discrepancy in the concept of improved/ worsened itself.

Another thing to keep in mind is that here we are in a somewhat strange prediction because we say forecast to have severe OSAS compared to non- severe OSAS: Here we have to see the variables related to them: ouch is correlated because the definition of OSAS is based on this.

So we had to see the variables related to $\mathrm{AHI}$ at $\mathrm{t} 1$.

So the ROC curve was used in order to find the cut off. 
It was found and there is a discriminatory capacity and specificity. Surely AHI is a risk factor but we have so much variability so we have a limitation in the accuracy of the evaluation of the value of Or and this is reflected in the table of predictions in which all in all the patients had an equal average age, they had many things identical, at most one comorbidity present. In summary, the variables do not affect the trend; a large share of imponderability remains.

There is nothing that systematically (according to statistics) can explain the errors. Something happened that changed the course, but this something is not explained by any of the variables considered.

\section{Trasversal study}

A population of known OSAS patients has been considered in order to evaluate if the presence or absence of therapy could affect the possibility of contracting Covid infection.

Several variables were considered as age, gender, therapy yes or not, comorbidities, AHI, therapy compliance.

The study shows that the ages in the two known groups of OSAS patients, those in therapy and that not in therapy, were not statistically significant.

Cardiovascular pathologies were by far the most represented (70\%) and most patients had at least one comorbidity (58\%); but between the two groups there was no statistically significant difference in the distribution of comorbidities and the number of concurrently present comorbidities.

$12 \%$ of treated OSAS patients developed Covid versus $16 \%$ of not treated OSAS patients with no significant differences in the two groups regarding the course of the disease.

There was no correlation between the variables studied and the development of the Covid.

Not even the severity of OSAS, determined by the $\mathrm{AHI}$, seems to have affected a greater chance of contracting the virus.

Finally, a comparison was made between patients without comorbidities and patients with at least one comorbidity: There were no significant differences in the cases of Covid in the two groups.

Summing up on 110 OSAS patients $20 \%$ developed Covid.

No significance is due to the low sample size.

This is because almost all of the patients followed by sleep lab follow the therapy; the maximum number of known OSAS patients not in therapy was 55 . The sample size affects the power of a test that is the ability to identify an effect when it exists.
Probably in this study a greater sample size is needed to determine the magnitude of the effect, that is the effect size.

It is interesting to ask whether the percentage of OSAS patients who took Covid is compatible with the distribution of the general population. So, is OSAS a risk factor?

In Veneto the Covid patients have been 426 out of 5584: the $7 \%$ of population vs. $14 \%$ of OSAS patients during February 2021.

\section{Conclusions}

Although sleep apnea syndrome causes an upper airway inflammatory condition, it is difficult to establish if that disease can promote Covid infection development.

The variables observed in a longitudinal study such as age, BMI, supine time, hours sleep, oxygen saturation, respiratory rate and comorbidities at T0 and T1 time were not statistically significant referring to OSAS evolution in Covid infection.

Comorbidities does not affect OSAS trend.

The trasversal study also does not find a significant correlation between the two groups of treated/not treated patients and Covid infection: $12 \%$ of treated OSAS patients developed Covid infection vs. $16 \%$ of not treated OSAS patients. It is important to underline that a small sample is a limit of this study.

That limit is caused by the fact that not treated OSAS patients in sleep laboratory are very few. Nevertheless, these Data suggests something and data collected from Pederzoli Hospital about general population in Veneto who developed Covid infection in February 2021 show they were the $7 \%$ vs. the $14 \%$ of OSAS population.

All data collection about OSAS patients is contemporaneous with the collection of non-OSA data of the general population in Veneto.

Sleep apnea syndrome is a Covid risk factor.

OSAS therapy could be a protective factor.

Most of literature data speaks about OSAS as negative prognostic factor to Covid patients hospitalization, some of them speaks about OSAS as a Covid risk factor.

No one of them reports how OSAS diagnosis was made, how the sleep study evaluation was done and if OSAS hospitalized Covid patients were or not in treatment and which is the therapy compliance; because there is a big difference between a treated OSAS patient and a treated OSAS patient with a low compliance; that one is like a not treated OSAS patient!

The strengths of this study are:

- Suitable sleep study device

- Correct sleep study analysis (manual scoring) 
- Expertise in efficacy therapy evaluating (considering using time of therapy during the night)

Limitations are referring to a limited evaluation period between $\mathrm{T} 0$ and $\mathrm{T} 1$, a difficulty in a greater data collection for the pandemic period, a low sample size, a lack of knowledge of Covid consequences duration and the fact that not treated OSAS patients were few (if a higher number of OSAS patients had refused the treatment we would have had a more reliable comparison with the group of patients in therapy).

\section{References}

1. Lombardi C, Pengo MF, Parati G (2018) Systemic hypertension in obstructive sleep apnea. J Thorac Dis 10: S4231-S4243.

2. Richardson S, Hirsch JS, Narasimhan M, Crawford JM, McGinn TM, et al. (2020) Presenting characteristics, comorbidities, and outcomes among 5700 patients hospitalized with COVID-19 in the New York City area. JAMA 323: 2052-2059.

3. Guan WJ, Ni ZY, Hu Y, Liang WH, Ou CQ, et al. (2020) Clinical characteristics of coronavirus disease 2019 in China. N Engl J Med 382: 1708-1720.
4. Gupta MA, Simpson FC (2015) Obstructive sleep apnea and psychiatric disorders: A systematic review. J Clin Sleep Med 11: 165-175.

5. Tietjens JR, Claman D, Kezirian EJ, De Marco T, Mirzayan A, et al. (2019) Obstructive sleep apnea in cardiovascular disease: A review of the literature and proposed multidisciplinary clinical management strategy. J Am Heart Assoc 8: e010440.

6. Reutrakul S, Mokhlesi B (2017) Obstructive sleep apnea and diabetes: A state of the art review. Chest 152: 10701086.

7. Wickramasinghe H (2020) Obstructive Sleep Apnea (OSAS).

8. Hou H, Zhao Y, Yu W, Dong H, Xue X, et al. (2018) Association of obstructive sleep apnea with hypertension: A systematic review and meta-analysis. J Glob Health 8: 010405 e.

9. Kheirandish-Gozal L, Gozal D (2019) Obstructive sleep apnea and inflammation: Proof of concept based on two illustrative cytokines. Int J Mol Sci 20: 459.

10. Patil SP, Ayappa IA, Caples SM, Kimoff RJ, Patel SR, et al. (2019) Treatment of adult obstructive sleep apnea with positive airway pressure: An american academy of sleep medicine systematic review, meta- analysis, and GRADE assessment. J Clin Sleep Med 15: 301-334. 